\title{
EVOLUTION OF WIRELESS MOBILE COMMUNICATION NETWORKS AND FUTURE OF CEllular MARKET IN INDia
}

\author{
Arjun Kondur ${ }^{1}$, Mayur Rao ${ }^{1}$, Pavan Kumar B $S^{1}$ and Rajeshwari Hegde ${ }^{1}$ \\ Dept of Telecommunication Engg, BMS College of Engineering, Bangalore, India \\ \{kondurarjun, mayurrao2801, pavanbs.kumar, rajeshwari.hegde\}@gmail.com
}

\begin{abstract}
In this paper, we discuss the evolution of the mobile communication systems from GSM to LTE (2G to 4G) and the trends in the mobile communication industry. The Global System for Mobile Communication $(G S M)$ is a well established cellular system targeted here due to its engineering success and the large number of users currently using the services. The flexibility of wireless networks over voice and data transmission makes it one of the most popular modes of communication. Evolution to next generation services depends on an addition of new services and new features to the existing networks or even an integration of different communication technologies. With the invention of the devices such as tablets and smart phones the need to improve the data transmission rates and transmission efficiency has increased to higher than ever before. In this paper, we focus at some of the important issues pertaining to the evolution of mobile communication networks and predict the future of the networks based on the analysis of the cellular market in India. Since GSM networks accounts for more than 75\% of the world wide cellular network, only the evolution of GSM network has been discussed in this paper.
\end{abstract}

\section{KEY WORDS}

GSM, EDGE, GPRS, UMTS, $4 G$

\section{INTRODUCTION}

The wireless technology has come a long way since its inception. There has been an impressive growth in the number of wireless users all over the world over the last decade. The use of wireless technology is now not just limited to wireless telephony but there is now a huge demand of wireless technology for many types of applications like Internet and web browsing, video and other text and multimedia based applications [1]. GSM is the most widely deployed $2^{\text {nd }}$ generation digital cellular standard, with over 3 billion subscribers in some 215 countries and adding about 1000 new users per minute. GSM which uses both TDMA and FDMA schemes, providing wide area voice communications using $200 \mathrm{KHz}$ carriers was originally developed in the 1980's and first deployed in 1991. Later, it evolved into $2.5 \mathrm{G}$ standards with the introduction of packet data transmission technology (GPRS) and higher data rates via higher order modulation schemes (EDGE). Together they are now referred to as GERAN, for GSM/EDGE based Radio Access Network [2]. Since the creation of the first truly global mobile telephony system - GSM in 1992, mobile communications have rapidly become an indispensable icon of our times. Some security features were built into the original GSM in order to ensure the privacy of user related information. GSM provides mobile phone communication services based on digital data interchange at up to $9.6 \mathrm{kbps}$, in addition to audio communication [3]. Most of the GSM standards were designed from an operator perspective with a view to preventing fraud and network misuse; the responsibility for implementing features related to user's privacy was delegated to the

Sundarapandian et al. (Eds): CoNeCo,WiMo, NLP, CRYPSIS, ICAIT, ICDIP, ITCSE, CS \& IT 07, pp. 453-462, 2012. () CS \& IT-CSCP 2012

DOI : $10.5121 /$ csit.2012.2444 
operators [4]. While constituting a substantial evolutionary step, the new modes such as GPRS, EDGE will fit well into the present GSM structure, and require only minimal changes to the network and base stations. It is of interest to consider how the evolutionary path can be extended to even higher bit rates, allowing for an even larger variety of services, and reaching the requirements of UMTS [5].There are drawbacks to the current GSM:

1) The GSM is a circuit-switched, connection-oriented technology, where the end systems are dedicated for the entire call session. This causes inaccuracy in usage of bandwidth and resources.

2) The GSM-enabled systems do not support high data rates [3].

3) The greatest disadvantage of GSM is that multiple users share the same bandwidth. With enough users, the transmission can encounter interference. Therefore, faster technologies, such as 3G, have been developed on different types of networks than GSM, such as CDMA, in order to avoid such bandwidth limitations.

4) Another disadvantage of GSM is that is can interfere with certain electronics devices used by human being, such as pace makers and hearing aids, according to Inc.Technology.com. Such interference is due to the fact that GSM uses a pulse-transmission technology. As a result, many locations such as hospitals and airplanes require cell phones to be turned off [6]. The roaming of cell phones for voice, the ability for a user to make and receive calls when visiting another country, is taken for granted by GSM users today. The GSM mobile operators have introduced packet-based data networks, General Packet Radio Service (GPRS) that brings a huge variety of services like video conferences, multimedia applications etc. However, this new network also introduces new complexity in the support of the packet-based roaming scenario. Due to the number of services, operators, and users envisaged, it will be impossible for operators to test all services for roaming in all partner networks for GPRS. Furthermore, mobile data service developers must be aware that the network capabilities of a visited GPRS network might differ from the home network; thus, consideration is required to ensure service continuity. This is especially true as mobile operators start offering $3 \mathrm{G}$ networks and support roaming agreements from $3 \mathrm{G}$ to $2 \mathrm{G}$ networks [7]. The paper is organized as follows. Section 2 explains GSM networks. Section 3 deals with Physical Layer Features of GSM,GPRS/EDGE,UMTS, LTE. Section 4 deals with the migration to 4G. Section 5 deals with GSM subscriber growth and technology trend in India. The paper is concluded in section 6.

\section{GSM NETWORKS}

The second generation networks deliver high-quality and secure mobile voice and basic data services such as fax and text messaging along with full roaming capabilities across the world. The basic GSM could originally provide only a 9.6-Kbps user data rate [11]. Hence the developments were made to upgrade $2 \mathrm{G}$ networks without replacing the networks to address the poor data transmission rates of the $2 \mathrm{G}$ networks. These technological advancements were called $2.5 \mathrm{G}$ technologies and include networks such as general GPRS. GPRS enabled networks deliver features such as always-on, higher capacity, internet based content and packet based data services, enabling services such as color internet service, email on the move, visual communications, multimedia messages and location based services. Another complimentary 2.5G service is enhanced data rules for GSM evolution (EDGE). This network upgrade offers similar capabilities as those of the GPRS networks. The High Speed Circuit Switched Data (HSCSD) is another 2.5G network enhancement of data services. This allows access to non-voice services three times faster than conventional networks, which means subscribers are able to send and receive data from the portable computers at speeds up to $28.8 \mathrm{Kbps}$ which is currently being upgraded in many networks to $43.2 \mathrm{Kbps}$. The HSCSD solution enables higher rates by using multiple channels, 
thereby allowing subscribers to enjoy faster rates for their internet, email, calendar and file transfer services.

Initially, $2 \mathrm{G}$ networks were created mainly for slow transmission and voice data. Because of the quick changes in the expectations of users, these no longer meet the requirements today in terms of wireless connection. Figure 1 shows the evolution to $3 \mathrm{G}$.



Figure 1. Evolution to $3 \mathrm{G}$

To overcome the drawbacks of $2 \mathrm{G}$ system, $3 \mathrm{G}$ systems were developed which is used to define an umbrella of standards and systems for next generation mobile system [12]. Fig. 2 shows the services offered by the $3 \mathrm{G}$ systems. Table 1 shows the technological advancements from $2 \mathrm{G}$ to $3 \mathrm{G}$ systems.

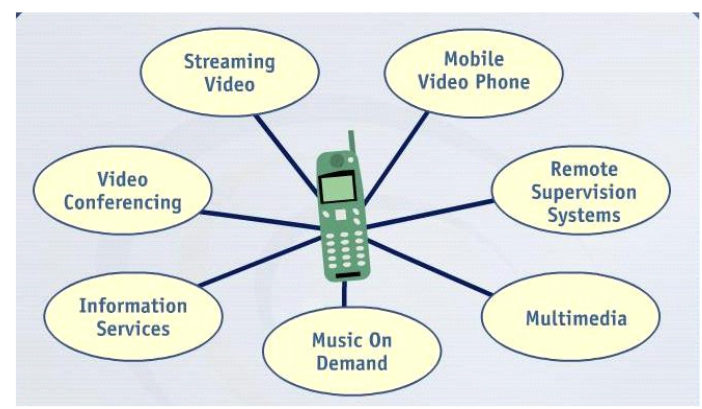

Figure 2. Capabilities of 3G

TABLE 1: Technological advancement from $2 \mathrm{G}$ to $3 \mathrm{G}$

\begin{tabular}{|l|l|l|l|}
\hline Technology & $\begin{array}{l}\text { Max } \\
\text { Data } \\
\text { Rate }\end{array}$ & Spectrum & Services \\
\hline GSM & $\begin{array}{l}57.6 \mathrm{~kb} \\
\text { ps }\end{array}$ & $200 \mathrm{kHz}$ & $\begin{array}{l}\text { Voice and circuit } \\
\text { data services }\end{array}$ \\
\hline GPRS & $\begin{array}{l}171.6 \mathrm{k} \\
\text { bps }\end{array}$ & $200 \mathrm{kHz}$ & $\begin{array}{l}\text { Packet data } \\
\text { services }\end{array}$ \\
\hline EDGE & $\begin{array}{l}473.6 \mathrm{k} \\
\text { bps }\end{array}$ & $200 \mathrm{kHz}$ & $\begin{array}{l}\text { Packet data } \\
\text { services }\end{array}$ \\
\hline UMTS & $2 \mathrm{Mbps}$ & $5 \mathrm{MHz}$ & $\begin{array}{l}\text { Voice and packet } \\
\text { data services }\end{array}$ \\
\hline
\end{tabular}

The following section explains the $3 \mathrm{G}$ cellular services. 


\subsection{General Packet Radio Service (GPRS)}

The first big step in the move to 3G happened through the launching of GPRS. The cellular services, mixed with GPRS resulted to $2.5 \mathrm{G}$. GPRS was capable of giving data rates ranging from $56 \mathrm{kbbps}$ up to a maximum of $114 \mathrm{kbps}$. This can be used for services like Wireless Application Protocol access, Multimedia Messaging Service (MMS), Short Message Service (SMS) and internet communication services like World Wide Web access and email. The data transfer of GPRS is usually charged for each megabyte of traffic being transferred, while the data communication via the usual circuit switching is charged by the minute of connection period, regardless of whether the consumer actually used the capability or is just in idle mode.

GPRS is a top-effort packet switched service, compared to circuit switching, where there is a given Quality of Service (QoS) is certified during the connection for non-mobile users. It gives medium speed data transfer, via the use of idle Time division multiple access (TDMA) channels.

\subsection{Enhanced Data rates for GSM Evolution (EDGE)}

Further enhancements to GSM network is provided by EDGE technology, which provides up to three times the data capacity of GPRS. Using EDGE, operators can handle three times more subscribers than GPRS, triple their data rate per subscriber, or add extra capacity to their voice communication. EDGE allows the delivery of advanced mobile services such as the downloading of video and music clips, multimedia messaging, high-speed Internet access and e-mail on the move [14]. EDGE is essentially a GSW GPRS radio interface with a set of enhancements to support higher peak data rates than GPRS and to provide better data throughput than GPRS. According to the US operators intending to use EDGE, it is mainly intended to provide 3G-type data services in a combined GSM and TIAIEIA-136 footprint in all of the existing 800/900/1800/1900 MHz frequency bands [16]. The GPRS networks have changed significantly to EDGE networks, through the presentation of 8PSK encoding. Enhanced information rates for EDGE or GSM Evolution, IMT Single Carrier or IMT-SC and Enhanced GPRS is a reversecompatible digital mobile phone technology, allowing improved data transmission rates, as an extension over the standard GSM. EDGE can be counted as a 3G radio technology, involved in ITU's $3 \mathrm{G}$ description, but is frequently referred to as $2.75 \mathrm{G}$. It was launched on GSM networks, starting in 2003, by Cingular. 3GPP standardized EDGE as it belonged in the GSM group. The specification gets bigger data rates by altering to very sophisticated processes of coding, particularly 8PSK, inside the GSM timeslots [1]. Fig. 3 shows the GPRS and EDGE architecture. GPRS is a $2.5 \mathrm{G}$ solution that provides medium speed packet data service for a wireless network.

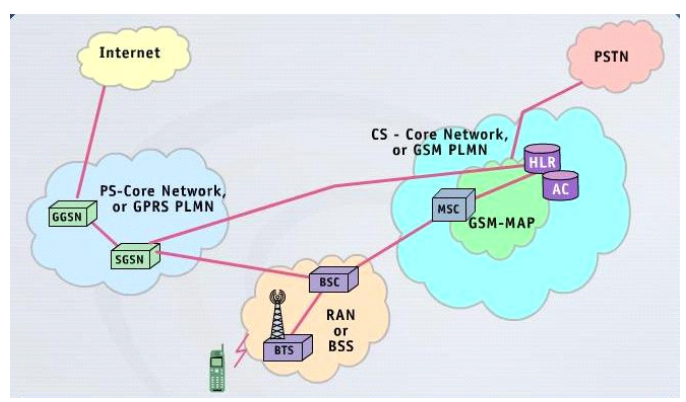

Figure 3. GPRS and EDGE architecture

\subsection{Universal Mobile Telecommunication Systems (UMTS)}

The most promising period is the advent of third generation $(3 \mathrm{G})$ networks which are also refer to as the UMTS. The global standardization effort undertaking by the ITU is called IMT-2000. The aim of the group was to evolve today's circuit switched core network to support new spectrum 
allocations and higher bandwidth capabilities. More than $85 \%$ of the world's network operators have chosen $3 \mathrm{G}$ as the underlying technology platform to deliver their third generation services.

UMTS which is the third generation system promoted by ETSI provides vital link between today's multiple GSM systems and the ultimate single worldwide system for all mobile telecommunications. New challenges in the design of $3 \mathrm{G}$ mobile networks have lead to a modification of the $2 \mathrm{G}$ system design. In order to reduce the network deployment costs, the instinctive approach for site acquisition is to re-use GSM cell locations as much as possible. Realtime services are confined to dedicated connections whereas non-real-time low-bandwidth services can quite easily use shared communication channels, which can more easily be changed dynamically [17].

\subsection{Long Term Evolution (LTE)}

The 3GPP LTE is a significant evolutionary step for UMTS in terms of capacity and architecture. It is designed to be backward compatible with GSM and HSPA. LTE incorporates Multiple In Multiple Out (MIMO) in combination with Orthogonal Frequency Division Multiple Access (OFDMA) in the downlink and Single Carrier FDMA in the uplink to provide high levels of spectral efficiency and end user data rates exceeding $100 \mathrm{Mbps}$, coupled with major improvements in capacity and reductions in latency. LTE supports channel bandwidths from 1.25 $\mathrm{MHz}$ to $20 \mathrm{MHz}$ and both FDD and TDD operation.

\section{Physical Layer Features of GSM,GPRS/EDGE,UMTS, LTE}

GSM access scheme is TDMA with eight basic physical channels per carrier with carrier separation of $200 \mathrm{kHz}$. A physical channel is therefore defined as a sequence of TDMA frames with 8 time slot/frame with 15/26 ms interval [15]. The air interface in case of GPRS radio interface consists of a number of protocol layers including the Radio Link Control (RLC), Medium Access Control (MAC) and physical GSM RF layers. GPRS uses the air interface provided by GSM physical layer. The physical layer, similar to GSM (GMSK), includes functions such as modulation/demodulation (GMSK), data coding and detection and correction of channel errors. The coding schemes CS-I to CS-4 have been defined for GPRS. GPRS phase 1 uses CS-1 and CS-2 coding that offer speeds of $9.05 \mathrm{kbps}$ and $13.4 \mathrm{kbps}$ per timeslot respectively. These speeds are suitable only for textual transmission of wireless internet services. However it requires no changes to a well-designed GSM networks. The coding schemes CS-3 and CS-4 offer speeds of $15.05 \mathrm{kbps}$ and $21.4 \mathrm{kbps}$ per timeslot respectively. These standard will require higher $\mathrm{C} / \mathrm{I}$ and higher signal level, hence coverage re-planning is necessary. The physical channels in GPRS are referred to as Packet Data Channel (PDCH). The corresponding mapped logical channels in GPRS have its own logical multiframe structure that is completely different from the existing structure used in GSM [19].

The EDGE introduces new methods at the physical layer including two new modulation schemes, Quaternary Offset Quadrature Amplitude Modulation Q-0-QAM and Binary Offset Quadrature Amplitude Modulation B-0-QAM ( instead of GMSK used in GSM) and different techniques of encoding data to protect against errors. These two new schemes will both result in symbol rates of $361.111 \mathrm{Kbps}$ however Q-0-QAM will offer a higher bit rate as it support 2bitlsymbol However higher layer protocols such as used in GGSN and SGSN will stay the same as in GPRS [19]. UMTS system physical channels are arranged into $10 \mathrm{~ms}$ radio frames which consist of 15 slots of $0.67 \mathrm{~ms}$ each. Each slot corresponds to 2560 chips or chip rate of $3.84 \mathrm{Mcps}$. UMTS uses a OVSF codes and Gold codes for spreading and scrambling respectively. There are 8192 available scrambling codes, arranged into 512 sets containing one primary and 15 secondary scrambling codes. 
The LTE PHY specification is designed to accommodate bandwidths from $1.25 \mathrm{MHz}$ to $20 \mathrm{MHz}$. OFDM was selected as the basic modulation scheme because of its robustness in the presence of severe multipath fading. Downlink multiplexing is accomplished via OFDMA. The DL supports physical channels, which convey information from higher layers in the LTE stack, and physical signals which are for the exclusive use of the PHY layer. Physical channels map to transport channels, which are Service Access Points (SAPs) for the L2/L3 layers. Depending on the assigned task, physical channels and signals use different modulation and coding parameters [20]. To support transmission in paired and unpaired spectrum, two duplex modes are supported: Frequency Division Duplex (FDD), supporting full duplex and half duplex operation, and Time Division Duplex (TDD).

\section{Migration to 4G Mobile System}

With the rapid development of wireless communication networks, it is expected that the 4 th generation mobile systems will be launched within decades. 4G mobile systems focus on seamlessly integrating the existing wireless technologies including GSM, wireless LAN, and Bluetooth. This contrasts with $3 \mathrm{G}$, which merely focuses on developing new standards and hardware. 4G systems will support comprehensive and personalized services, providing stable system performance and quality service [21]. 4G is future technology for mobile and wireless communications.

\section{GSM SUBSCRIBER GROWTH AND TECHNOLOGY TREND IN INDIA}

The technologies of mobile telecommunications and Internet are going to set the contours of further technological progress in the current decade and the next. The most recent initiative aims at convergence of voice and data received from multiple sources, both web based and real time video streams, in mobile handheld devices. The Global satellite systems, mobile handsets and calling cards have made virtual presence possible almost everywhere and anywhere overcoming the barriers of distance, topography and remoteness.

There has been a phenomenal growth in mobile subscribers in the world in the nineties, increasing from 11 million in 1990 to 941 million by the end of 2001. In 1991, less than one per cent of the world population had a mobile phone. The proportion has grown to the vicinity of one phone per every six people by the end of 2001. Similarly, one-third of the total number of countries of the world had cellular network in 1991. The ratio rose to over 90 per cent by end-2001. Considering that the fixed telephone lines numbered just over a billion in this year, it is likely that mobile phones would surpass fixed line in 2002. It is interesting to observe that China has surpassed USA to become the largest mobile market of the world. In Africa, mobile subscribers outnumber fixed line subscribers in more than half the countries. Mobile telephony has emerged as the major growth driver in this sector. But for expansion in mobile network, there would have been hardly any growth in telecommunications in many countries. In developed countries, mobile phones have complemented fixed lines whereas in many developing countries with low-level fixed line penetration, mobile has already surpassed fixed lines filling up supply gaps created due to inadequate growth in the latter. It has been observed that 'the ability of a country to grow its mobile network to the point where it overtakes the fixed-line network is not a function of its wealth...the crossover point can come as low as a fixed teledensity of 0.4 (for instance, in Malwai) to as high as 75 (the case of Luxembourg) and at any point in between.

There are three important economic implications of mobile explosion for the developing countries. First, by offering a viable techno-economic alternative it is helping in improving telecom penetration bypassing shortages of fixed lines. Consequently, it is bringing along with it all concomitant economic benefits of enhanced telecom accessibility. Second, it is promoting a better entrepreneurial culture and supporting employment generation through proliferation of 
kiosks. Third, there has been a shift in investment burden from state to private sector and the consumers.

Cellular mobile telephones subscribers in India increased from 77 thousand in 1995 to 3.6 million in 2000. By March 2002, it has grown to 6.4 million. Cellular subscribers in proportion to total number of telephone subscribers (basic plus cellular) has increased from 0.6 percent in 1995 to 14.6 percent in 2002. This is still lower than the average of 24.6 percent achieved by the lowincome countries in 2001. The corresponding ratio for lower middle-income countries is 41.8 percent, 52.8 percent for upper middle-income countries and 50.2 percent for high-income countries. India is yet to experience mobile explosion of the scale other countries have seen. One would expect a rapid growth in mobile telephony in coming decades. India has also achieved significant quality up gradation of its network in the 90s. Digital lines in proportion to total number of main telephone lines have increased from 87 per cent in 1995 to 99.8 percent in 1999 [22]. Fig. 4 and 5 indicate the number of subscribers in India in 2011 and 2012.

Number of Subscribers as of August 2011 (in millions)

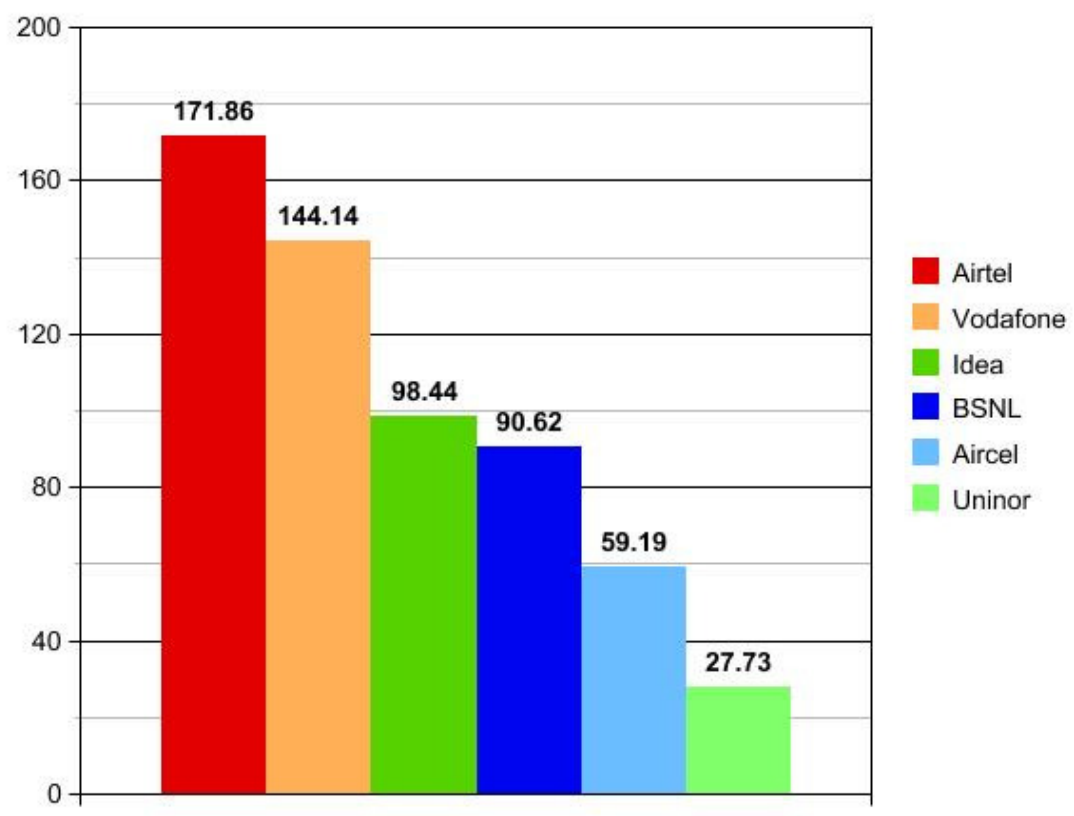

Figure 4. Company wise market share (Subscribers) as of August 2011 Total number of subscribers (approximate) in $2011=591.98$ million (source: Cellular Operators Association of India) 




Figure 5. Company wise market share (Subscribers) as of August 2012

Total number of subscribers (approximate) in 2012 $=658.96$ million [23]

The growth in number of subscribers from 2011 to 2012 is almost 67 million. This is an 11.3 percent increase in number of subscribers throughout India. This substantial increase in number of subscribers during a short span of 12 months gives us a general idea about the rate at which cellular growth, mainly GSM is developing in India. India currently ranks second behind China in largest number of mobile subscribers and second in largest mobile networks. Indian telecommunication Industry is the world's fastest growing industry with India possessing the second largest telecommunication network after China according to the recent survey by TRAI [24].

\section{CONCLuSiON}

This paper attempts to address some of the important issues pertaining to the migration from the most widely deployed $2^{\text {nd }}$ generation digital cellular system namely GSM to $4 \mathrm{G}$ in India. The drift from GSM to UMTS is both pragmatic and imminent. The rising demand for high quality services by the customers and providing cost effective services by the operators has been due to the ability to roam globally, huge economies of scale, widespread acceptance by operators, complementary services such as multimedia messaging and a wide variety of competitive handsets. With support from nearly all major regional standardization bodies UMTS is already the world's most selected 3rd generation technology. The stepping stone towards the evolution of GSM to UMTS is GPRS, an IP-based packet-data capability for GSM networks which offers specific benefits and features. Going by the above statistics shown for the last two years, India has had phenomenal growth in the number of subscribers with different companies. Going by this growth we can expect the total number of subscribers in 2013 to be approximately 1 Billion. EDGE provides a cost effective 3G solution for operators to upgrade to an ITU-approved $3 \mathrm{G}$ technology which was not provided by GPRS. EDGE promises to be one of the most spectrally efficient technologies available for cellular data services. The introduction of $4 \mathrm{G}$ also comes with cost and implementation drawbacks and problems, especially in a country such as India. As demand for data services grows, operators can deploy UMTS networks, which support high bandwidth applications. India is likely to become world's leading mobile market by 2013 with more than a billion mobile users according to a recent survey by the Telecom Regulatory Authority Of India (TRAI). The global mobile application industry is expected to burgeon till $\$ 17.5$ billion by the end of this year according to recent surveys and reports. 


\section{REFERENCES}

[1] Azim Samjani, "General Packet Radio Service (GPRS)”, IEEE Potentials 2002, 2nd Edition, 2002:pp $10-13$.

[2] Bjorn Gudmundson, Johan Skold, Per-Olof Anderson, "Evolution of the GSM Air Interface into Wideband Services", The institution of Electrical Engineers, IEE 1996.

[3] Chen, YP; Yang." new 4G architecture providing multimode terminals always best connected services", IEEE Wireless Communications, Volume: 14 Issue: 2 pp. 36-41.

[4] C M H Noblet', R H Owen', C Saraiva2, N VJahid, “Assessing the Effects of GSM Cell Location Re-Use for UMTS Network", IEEE 2001.

[5] Dr. Monira A. Abu El-Ata, "Evolution of Mobile Cellular Communication Systems The Journey to UMTS”, 17thNational Radio Science Conference, NRSC'2000.

[6] Frattasi, S; Gimmler, " Potentials and limits of cooperation in wireless communications: Toward 4G wireless”, IEEE Technology and Society Magazine, Volume: 27 Issue: 1 pp. 8-12.

[7] G Kalimani Venkatesan, Kishor Kulkarni.: Wireless Backhaul for LTE - Requirements, Challenges and Options. IEEE 2nd International Symposium on Advanced Networks and Telecommunication Systems, 2008.

[8] Harri Holma , Antti , WCDMA for UMTS - HSPA Evolution and LTE, fourth edition. Toskala 2007 John Wiley \& Sons, Ltd.

[9] Hendra Setiawan, Hiroshi Ochi," Study Feasibility of Common Wireless Communication Services Recognition for GSM, UMTS and LTE”, The IEEE 2009 International Conference on Advanced Technologies for Communications.

[10] Jim Zyren. Overview of the 3GPP Long Term Evolution Physical Layer, Available at: http://www.freescale.com/files/wireless_comm/doc/white_paper/3GPPEVOLUTIONWP.pdf

[11] Joha Korhonen, Text book on "Introduction to 3G Mobile Communications", Artech House, Boston

[12] Ms. Shilpa Jindal Ms. Alka Jindal Dr. (Mrs.) Neena Gupta, "Grouping WI-MAX, 3G and WI-Ft for Wireless Broadband", IEEE 2005.

[13] Prabhakar Chitrapu, Behrouz Aghili, "Evoluton of GSM into the Next Generation Wireless World", Write the conference name (In italic), pp IEEE 2007.

[14] Qi Bi, George I. Zysman, and Hank Menkes," Wireless Mobile Communications at the Start of the 21st Century", IEEE Communications Magazine , January 2001

[15] Rao Yallapragada, Vera Kripalani and Ani1 Kripalani, "EDGE: A Technology Assessment”, ,

[16] S Gindraux, "From 2G to 3G: A Guide to Mobile Security. 3G Mobile Communication Technologies", Conference Publication No. 489, May 2002.

[17] Satyajit H. Rai, "GPRS and 3G Wireless Systems", Seminar report, Avaialble at: http://www.it.iitb.ac.in/ satyajit/seminar/seminar.pdf

[18] Suk Yu Hui and Kai Hau Yeung, "Challenges in the Migration to 4G Mobile Systems", IEEE Communications Magazine, December 2003.

[19] The Advantages and Disadvantages of GSM, Available at: http://www.ehow.com/list_6322410_advantages-disadvantages-gsm.html

[20] What is EDGE? http://www.gsmworld.com/technology/edge.htm

[21] Y.S. Rao, Wing-Cheong Yeung, and Ani1 Kripalani, "Third-Generation (3G) Radio Access Standards", IEEE 2000.

[22] Manas Bhattacharya , "Telecom Sector in India: Vision 2020", Background Paper submitted to the Committee on India: Vision 2020, available at:

[23] Company wise market share (Subscribers) as of August 2011, 2012 available at http://www.coai.com/statistics.php.

[24] Indian future scenario available at: http://www.dailypioneer.com/avenues/77726-india-is-biggestmobile-market.html. 


\section{Authors}

1.Arjun Kondur is currently pursuing his B.E at the Dept. of Telecommunications Engineering, BMS College of Engineering, Bangalore. He is pursuing his final semester project at the Indian Institute of Science, Bangalore. He has a placement offer at Ericsson. $\mathrm{He}$ has done two internships at Bell Teleservices and Tata Communications Limited.He plans on doing an M.S in Electrical Engineering abroad.

2.Mayur Rao is currently pursuing his B.E at the Dept. of Telecommunication Engineering, BMS College of Engineering, Bangalore. He has completed an internship at Tejas Networks Ltd., where he worked with FPGA design and development. He is currently working on his final year project based on Multi-Core Processors implemented using an FPGA. He aspires to pursue his M.S in Computer Engineering.

3.Pavan Kumar BS is currently pursuing his B.E at the department of Telecommunications Engineering, BMS College of Engineering, Bangalore. He is currently pursuing his final year project at the Indian Institute of Science, Bangalore. He has a placement offer at $\mathrm{Mu}$ Sigma. He aspires to pursue M.S and Phd.

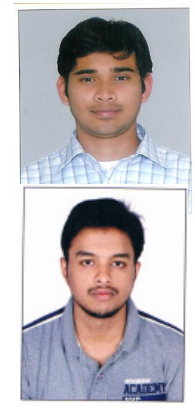

4.Rajeshwari Hegde is currently an Associate professor at the Dept. of Telecommunications Engineering, BMS College of Engineering, Bangalore. She received her M.E (Electronics) from BMS College of Engineering, Bangalore and B.E in Electronics and Communication Engineering from National Institute of Engineering, Mysore. She completed her $\mathrm{PhD}$ under the guidance of Dr K S Gurumurthy at UVCE, Bangalore University. She has published 47 research papers in international conferences, national conferences and reputed journals.



